International Journal of Distributed and Parallel Systems (IJDPS) Vol.3, No.4, July 2012

\title{
COMPARATIVE ANALYSIS OF RECTANGULAR AND CIRCULAR WAVEGUIDE USING MATLAB SIMULATION
}

\author{
AZHAR SHADAB ${ }^{1}$, LOKESH KUMAR $^{2}$, MOHIT KUMAR ${ }^{3}$, KAMAL KISHOR, $^{4}$ \\ AKASH SETHI $^{5}$, ILA SHARMA ${ }^{6}$ \\ ${ }^{1}$ Department of Electronics \& Communication, Jaypee University, Solan, \\ Himachal Pradesh, India \\ azharshadab786@gmail.com \\ ${ }^{2}$ Department of Electronics \& Communication, Jaypee University, Solan, \\ Himachal Pradesh, India \\ lokesh. chandravanshi@gmail.com \\ ${ }^{3}$ Department of Computer Science, Jaypee university, Solan, Himachal Pradesh, India \\ mohitsmailbox13@gmail.com \\ ${ }^{4}$ Department of Electronics \& Communication, Jaypee University, Solan, \\ Himachal Pradesh, India \\ KkC_43@yahoo.com \\ ${ }^{5}$ Department of Electronics \& Communication, Jaypee University, Solan, \\ Himachal Pradesh, India \\ akashsethi0910@gmail.com \\ ${ }^{6}$ Department of Electronics \& Communication, Jaypee University, Solan, \\ Himachal Pradesh, India \\ ilasharma23@gmail.com
}

\begin{abstract}
Electromagnetic waves is very strong waves which is used for transmission of signals from one place to other, main concern is medium having some physical structure through which it can propagate their waves. There are so many ways to guide this electromagnetic waves but main consideration in this paper is rectangular waveguide and circular waveguide.This paper analyze the different parameters of both the waveguides like (Cut off frequency, Cut off wavelength,Guide wavelength,Phase velocity, Group velocity, Impedance, power, Attenuation, VSWR) with the help of simulation in Matlab7.14 .Compare the parameters of the two waveguides and on the basis of these parameters result it is easy to get the best guided structure.
\end{abstract}

Keywords: Rectangular waveguide, Circular waveguide, Waveguide modes, Matlab simulation.

\section{Introduction}

The history of transmission originated from the work of JAMES CLERK MAXWELL, LORD KELVIN and OLIVER HEAVISIDE. Now a day for transmitting the waves from one place to

DOI : $10.5121 /$ ijdps.2012.3405 
International Journal of Distributed and Parallel Systems (IJDPS) Vol.3, No.4, July 2012

another there are so many different types of structures are used. Some of the structures are as popularly known as wires, coaxial cables, dielectric slabs, strip lines, optical fibers, electric power lines, and waveguides. This paper described for rectangular waveguides and circular waveguides.It consist of some modes and on the basis of that modes its operation is based.There are basically three types of modes which are as follows:

- TE modes (Transverse Electric) no electric field in the direction of propagation.

- TM modes (Transverse Magnetic mode) in the direction of propagation there is no magnetic field.

- TEM modes (Transverse Electro Magnetic) neither electric nor magnetic field in the direction of propagation.

This paper generally consider $\mathrm{X}$ band for the range of frequency 8.2 to 12.4 GHZ.Both the waveguides having many different parameters which may distinguish to each other.On the basis of simulation in Matlab it is easy to understand the practical observation of these parameters .So it is easy to achieve the best guide medium of waves and these waveguide can be used in different practical purposes[1][2].

\section{Modes of Operating}

A rectangular waveguide supports TM and TE modes but not TEM modes because it cannot define a unique voltage since there is only one conductor in a rectangular waveguide. The shape of a rectangular waveguide based on their dimensions. A material with permittivity (e )and permeability $(\mathrm{m})$ fills the inside of the conductor.The mode in which the magnetic field is always transverse in the direction of propagation is called the Transverse magnetic (TM) mode,In Tm mode $\mathrm{H}_{\mathrm{z}}=0 \& \mathrm{E}_{\mathrm{z}} \neq 0$. The mode in which the electric field is always transverse to the direction of propagation is called the Transverse electric mode.Thus in TE mode $\mathrm{E}_{\mathrm{Z}}=0 \&$ $\mathrm{H}_{\mathrm{Z}} \neq 0$.

\subsection{For TM mode}

Consider the shape of the rectangular waveguide above with dimensions $a$ and $b$ (assume $a>b$ ) and the parameters (e) and (m) . For TM waves $\mathrm{H}_{z}=0$ and $\mathrm{E}_{\mathrm{z}}$ should be solved from equation for TM mode;

$\tilde{\mathrm{N}}_{\mathrm{xy}}^{2} \mathrm{E}_{\mathrm{z}}^{0}+\mathrm{h}^{2} \mathrm{E}_{\mathrm{z}}^{0}=0$

Since $E_{z}(x, y, z)=E_{z}^{0}(x, y) e^{-g z}$, getting equation,

$$
\left(\frac{\partial^{2}}{\partial x^{2}}+\frac{\partial^{2}}{\partial y^{2}}+h^{2}\right) E_{z}^{0}(x, y)=0
$$

If we use the method of separation of variables, that is $E_{z}{ }^{0}(x, y)=X(x) . Y(y)$ we get,

$$
-\frac{1}{X(x)} \frac{d^{2} X(x)}{d x^{2}}=\frac{1}{Y(y)} \frac{d^{2} Y(y)}{d y^{2}}+h^{2}
$$


As the equation contains $\mathrm{x}$ and $\mathrm{y}$ terms, these terms having equal constant value i.e $\mathrm{k}_{\mathrm{x}}{ }^{2}$

$$
\begin{aligned}
& \frac{d^{2} X(x)}{d x^{2}}+k_{x}^{2} X(x)=0 \\
& \frac{d^{2} Y(y)}{d y^{2}}+k_{y}^{2} Y(y)=0
\end{aligned}
$$

Where $\mathrm{k}_{\mathrm{y}}{ }^{2}=\mathrm{h}^{2}-\mathrm{k}_{\mathrm{x}}{ }^{2}$, Now solve for $\mathrm{X}$ and $\mathrm{Y}$ from the preceding equations.But there is some boundary condition[3] :

$\mathrm{E}_{\mathrm{z}}^{0}(0, \mathrm{y})=0, \mathrm{E}_{\mathrm{z}}^{0}(\mathrm{a}, \mathrm{y})=0, \mathrm{E}_{\mathrm{z}}^{0}(\mathrm{x}, 0)=0, \mathrm{E}_{\mathrm{z}}^{0}(\mathrm{x}, \mathrm{b})=0$

Let $\mathrm{X}(\mathrm{x})$ be the form of $\sin \mathrm{k}_{\mathrm{x}} \mathrm{x}$, now value of $\mathrm{k}_{\mathrm{x}}$ would be "mp/a" where $\mathrm{m}=1,2,3 \ldots \ldots$ and $\mathrm{Y}(\mathrm{y})$ be the form of $\sin \mathrm{k}_{\mathrm{y}} \mathrm{y}$ and also the value of $\mathrm{k}_{\mathrm{y}}$ would be "np/b" where $\mathrm{n}=1,2,3 \ldots \ldots$ So that the solution for the value of $E_{z}^{0}(x, y)$ is $E_{z}^{0}(x, y)=E_{0} \sin \left(\frac{m \pi}{a} x\right) \sin \left(\frac{n \pi}{b} y\right)(\mathrm{V} / \mathrm{m})$

From $\mathrm{k}_{\mathrm{y}}{ }^{2}=\mathrm{h}^{2}-\mathrm{k}_{\mathrm{x}}{ }^{2}$, we have;

$h^{2}=\left(\frac{m \pi}{a}\right)^{2}+\left(\frac{n \pi}{b}\right)^{2}$

For TM waves,

$$
\begin{aligned}
& H_{x}^{0}=\frac{j \omega \varepsilon}{h^{2}} \frac{\partial E_{z}^{0}}{\partial y} \\
& H_{y}^{0}=-\frac{j \omega \varepsilon}{h^{2}} \frac{\partial E_{z}^{0}}{\partial x} \\
& E_{x}^{0}=-\frac{\gamma}{h^{2}} \frac{\partial E_{z}^{0}}{\partial x} \\
& E_{y}^{0}=-\frac{\gamma}{h^{2}} \frac{\partial E_{z}^{0}}{\partial y}
\end{aligned}
$$

From above equations, getting equations 
International Journal of Distributed and Parallel Systems (IJDPS) Vol.3, No.4, July 2012

$$
\begin{gathered}
E_{x}^{0}(x, y)=-\frac{\gamma}{h^{2}}\left(\frac{m \pi}{a}\right) E_{0} \cos \left(\frac{m \pi}{a} x\right) \sin \left(\frac{n \pi}{b} y\right) \\
E_{y}^{0}(x, y)=-\frac{\gamma}{h^{2}}\left(\frac{n \pi}{b}\right) E_{0} \sin \left(\frac{m \pi}{a} x\right) \cos \left(\frac{n \pi}{b} y\right) \\
H_{x}^{0}(x, y)=\frac{j \omega \varepsilon}{h^{2}}\left(\frac{n \pi}{b}\right) E_{0} \sin \left(\frac{m \pi}{a} x\right) \cos \left(\frac{n \pi}{b} y\right) \\
H_{y}^{0}(x, y)=-\frac{j \omega \varepsilon}{h^{2}}\left(\frac{m \pi}{a}\right) E_{0} \cos \left(\frac{m \pi}{a} x\right) \sin \left(\frac{n \pi}{b} y\right)
\end{gathered}
$$

From the analyzation of above equations in the TM modes of rectangular waveguide, the values of $m \neq 0$ and $n \neq 0$. This is because the field expressions are identically zero, if the value of $m$ $=0$ or $\mathrm{n}=0$ [6].So the rectangular waveguide only deals with the TM mode having $\mathrm{m}=1$ and $\mathrm{n}=1$.

On the basis of different values of ' $m$ ' \&'n', there are different modes of $\mathrm{TM}_{\mathrm{mn}}[1][2]$ :

$\mathrm{TM}_{00}$ mode : If $\mathrm{m}=0$ \& $\mathrm{n}=0$ are substituted in $\mathrm{Ex}, \mathrm{Ey}, \mathrm{Hx}, \mathrm{Hy}$, then all of them vanish and hence $\mathrm{TM}_{00}$ mode cannot exist.

$\mathrm{TM}_{01}$ mode : If $\mathrm{m}=0 \& \mathrm{n}=1$ again all field components vanish and $\mathrm{TM}_{01}$ mode cannot exist.

$\mathrm{TM}_{10}$ mode : If $\mathrm{m}=1 \& \mathrm{n}=0$ all the field component vanishes hence $\mathrm{TM}_{10}$ mode cannot exist.

$\mathrm{TM}_{11}$ mode : There are all the four components Ex, Ey, Hx, Hy hence $\mathrm{TM}_{11}$ mode exists for all

higher values of $m \& n$.

\subsection{For TE Modes}

Consider again the rectangular waveguide below with dimensions $a$ and $b$ (assume $a>b$ ) and the parameters (e) and (m).For TE waves $E_{z}=0$ and $H_{z}$ should be solved from equation for TE mode;

$\tilde{\mathrm{N}}_{\mathrm{xy}}^{2} \mathrm{H}_{\mathrm{z}}+\mathrm{h}^{2} \mathrm{H}_{\mathrm{z}}=0$ Since $\mathrm{H}_{\mathrm{z}}(\mathrm{x}, \mathrm{y}, \mathrm{z})=\mathrm{H}_{\mathrm{z}}{ }^{0}(\mathrm{x}, \mathrm{y}) \mathrm{e}^{-\mathrm{gz}}$, getting equation,

$$
\left(\frac{\partial^{2}}{\partial x^{2}}+\frac{\partial^{2}}{\partial y^{2}}+h^{2}\right) H_{Z}^{0}(x, y)=0
$$

If using the method of separation of variables, that is $\mathrm{H}_{\mathrm{z}}{ }^{0}(\mathrm{x}, \mathrm{y})=\mathrm{X}(\mathrm{x}) . \mathrm{Y}(\mathrm{y})$ then,

$$
-\frac{1}{X(x)} \frac{d^{2} X(x)}{d x^{2}}=\frac{1}{Y(y)} \frac{d^{2} Y(y)}{d y^{2}}+h^{2}
$$

As the equation contains $\mathrm{x}$ and $\mathrm{y}$ terms, these terms having equal constant value i.e $\mathrm{k}_{\mathrm{x}}{ }^{2}$ 


$$
\begin{aligned}
& \frac{d^{2} X(x)}{d x^{2}}+k_{x}^{2} X(x)=0 \\
& \frac{d^{2} Y(y)}{d y^{2}}+k_{y}^{2} Y(y)=0
\end{aligned}
$$

Where $\mathrm{k}_{\mathrm{y}}^{2}=\mathrm{h}^{2}-\mathrm{k}_{\mathrm{x}}^{2}$

Solving for $\mathrm{X}$ and $\mathrm{Y}$ from the preceding equations. Also there is boundary condition:

$$
\begin{aligned}
& H_{z}^{0}(x, y)=H_{0} \cos \left(\frac{m \pi}{a} x\right) \cos \left(\frac{n \pi}{b} y\right) \\
& h^{2}=\left(\frac{m \pi}{a}\right)^{2}+\left(\frac{n \pi}{b}\right)^{2}
\end{aligned}
$$

The different possible modes is based on the different values of ' $m$ ' and ' $n$ ' This paper shows the $\mathrm{TE}_{\mathrm{mn}}$ mode.In the $\mathrm{x}$ and $\mathrm{y}$ direction of the propagation, ' $\mathrm{m}$ ' and ' $\mathrm{n}$ ' shows the no. of $1 / 2$ cycle variations of the fields respectively.

\subsection{Dominant Mode}

This mode is one of the most efficient mode and all the waveguides are designed in such a way that only this mode will be used.To operate in the dominant mode, a waveguide must have an "a" (wide) dimension of at least one half-wavelength of the frequency to be propagated.For the possible modes of operation available for a given waveguide, the dominant mode has the lowest cutoff frequency.In practice, this dimension is usually 0.7 wavelength[1].

$$
\lambda_{c}=\frac{2}{\sqrt{\left(\frac{m}{a}\right)^{2}+\left(\frac{n}{b}\right)^{2}}}(m)
$$

\section{Circular Waveguide}

Circular waveguide is basically seems like the circular structure where it consist tubular circular conductor.As the frequency of transmitted signal changes the inner diameter of circular waveguide also changes.There are so many uses of Circular waveguides like in communication systems used in specific areas of radar and uses as rotating joints of the mechanical point of the antennas rotation.Generally the values of radius is very important for manufacturing of circular waveguides.

\subsection{Modes of circular waveguide}

Modes of the circular waveguide shows that the Electric field is perpendicular to the length of waveguide and there is no any Electric lines parallel to the direction of propagation.The 
International Journal of Distributed and Parallel Systems (IJDPS) Vol.3, No.4, July 2012

dominant mode of the circular waveguide is $\mathrm{TE}_{11}$ where $\mathrm{m}=1$ and $\mathrm{n}=1$.For complete one cycle ' $m$ ' and ' $n$ ' shows the no. of $1 / 2$ cycle variations of the fields along the diameter.In this mode,waveguide shows the lowest cut off frequency required for operating. The cutoff wavelength of a circular guide is 1.71 times the diameter of the waveguide.In the circular waveguide,the $\mathrm{E}$ field is perpendicular to the length of the waveguide with no E lines parallel to the direction of propagation.Thus, it must be classified as operating in the TE mode.

\section{Related work}

This paper analyze the each parameters of the rectangular and the circular waveguide which help to select the waveguide medium according to the requirement.But from the ideas of the different papers of different authors it is clear that no any author is compared the parameters of these two waveguides, They only consider some parameters of either rectangular or circular waveguide but there is no any simulation part based on Matlab were used by any other authors.This paper give the complete details of each parameters with their mathematical calculations and their simulation results.

\section{Comparative view of modes of waveguide}


Figure 1. For rectangular waveguide


Figure 2. For circular waveguide 
International Journal of Distributed and Parallel Systems (IJDPS) Vol.3, No.4, July 2012
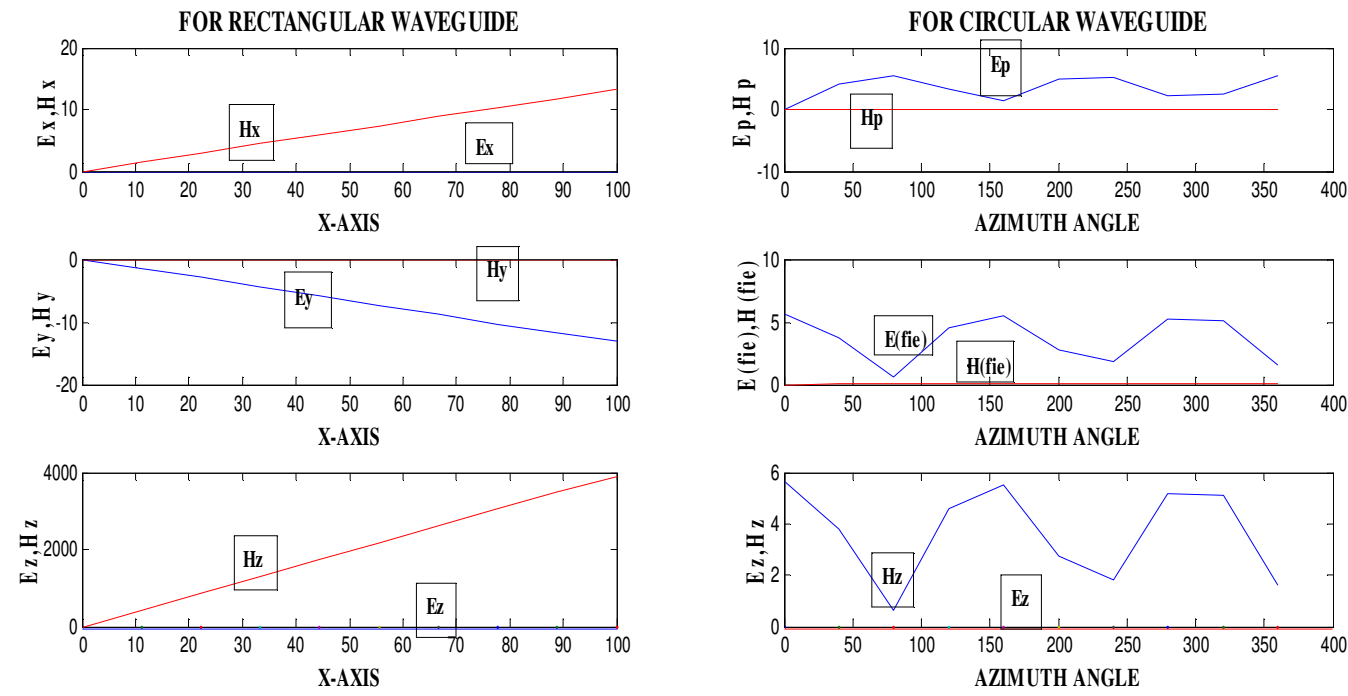

Figure 3. Rectangular waveguide



Figure 4. Circular waveguide

\section{Compared parameters content}

Cut off frequency,Cut off wavelength,Guide wavelength,Phase velocity ,Power \{(a)powwer transmission, (b)power losses, (c)break down power, (d)power handling capacity , Attenuation, VSWR

\subsection{Cut off Frequency}

Cut-off frequency is the frequency level from which the waves can start to propagate in the direction of field.These waveguides cannot propagate below that frequency level called as cut off frequency.The cutoff frequency is found with the characteristic equation of the Helmholtz equation for electromagnetic waves, which is derived from the electromagnetic wave equation by setting the longitudinal wave number equal to zero and solving for the frequency. Thus, any exciting frequency lowers than the cutoff frequency will attenuate, rather than propagate. The cut off frequency is given by:

$f_{c}=\frac{1}{2 \sqrt{\varepsilon \mu}} \sqrt{\left(\frac{m}{z}\right)^{2}+\left(\frac{m}{z}\right)^{2}}(H z)$

\subsection{Cut off Wavelength}

The cut off wavelength is defined as the maximum wavelength of the waves to be transmitted through the waveguide. All the wavelengths greater than $\lambda_{c}$ are attenuated and those which is less than $\lambda_{\mathrm{c}}$ are allowed to propagate inside the waveguide.

The cut off wavelength is denoted as:

$\lambda_{c}=\frac{2}{\sqrt{\left(\frac{m}{a}\right)^{2}+\left(\frac{n}{b}\right)^{2}}}(m)$ 
International Journal of Distributed and Parallel Systems (IJDPS) Vol.3, No.4, July 2012

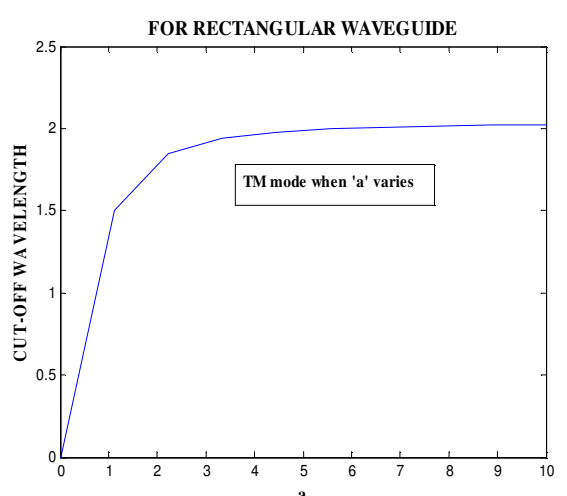

Figure 5. Rectangular waveguide in TM mode



Figure 7. Circular waveguide in TM mode

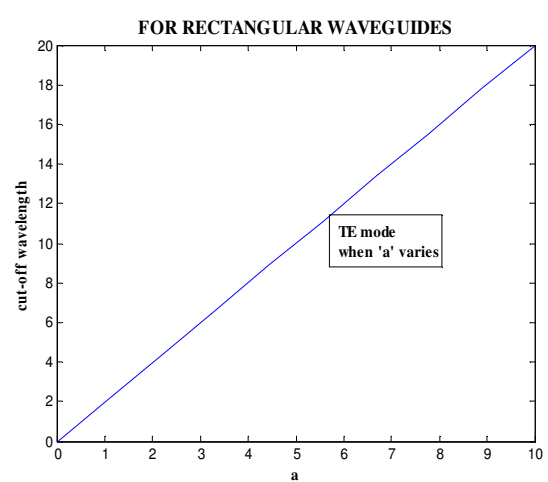

Figure 6. Rectangular waveguide in TE mode



Figure 8. Circular waveguide in TE mode

In circular waveguide the cut off wavelength is maximum when power is minimum in its different modes of TE and TM. But in rectangular waveguide the cut off wavelength rises for some point and then gets saturated at certain level of wavelength being unaware of power in the waveguide.

\subsection{Guide Wavelength}

The guide wavelength is defined as the distance between two equal phase planes along the waveguide. It is defined as the distance travelled by the wave in order to undergo a phase shift of $2 \pi$.

It is given as:

$\lambda_{g}=\frac{2 \pi}{\beta}$

where $\lambda_{g}=\lambda_{o} / \sqrt{ } 1-\left(\lambda_{o} / \lambda_{c}\right)^{\wedge} 2$ 
International Journal of Distributed and Parallel Systems (IJDPS) Vol.3, No.4, July 2012

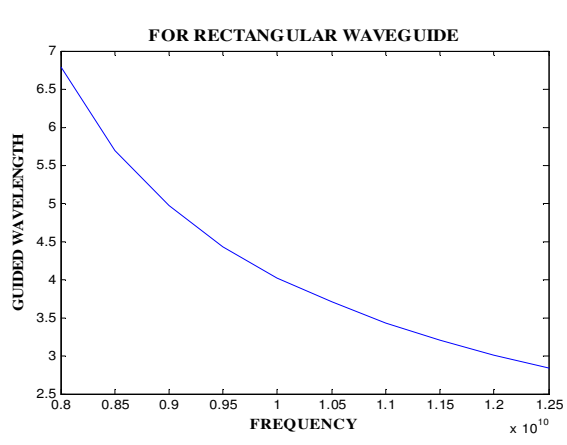

Figure 9 . Guided wavelength (Rectangular)



Figure 10 . Guided wavelength (Circular)

\subsection{Phase Velocity}

Phase Velocity is defined as the rate at which the wave changes its phase in terms of the guide wavelength.The wave propagates in the waveguide when guide wavelength $\lambda \mathrm{g}$ is greater than the free space wavelength $\lambda_{\mathrm{o}}$. Since the velocity of propagation is the product of $\lambda$ and $\mathrm{f}$, it follows that in a waveguide, $\mathrm{Vp}=\lambda \times f$ where $\mathrm{Vp}$ is the phase velocity. But $\mathrm{Vp}$ is greater than the speed of light since $\lambda \mathrm{g}>\lambda \mathrm{o}$. This is contradicting since no signal travels faster than speed of light. However the wavelength in the guide is the length of the cycle and Vp represents the velocity of the phase.

The phase velocity is given as;

$$
v_{p}=\frac{c}{\sqrt{1-\left(\frac{\omega_{0}}{\omega}\right)^{2}}}
$$

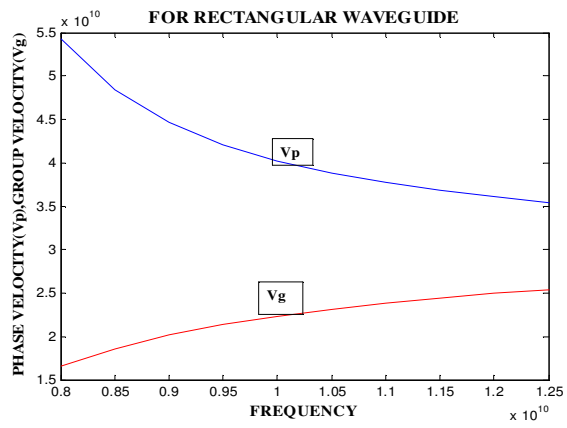

Figure 11. Phase velocity( Rectangular)

FOR RECTANGULAR WAVEGUIDE

For TE MODE: $Z_{T E}=\frac{k \eta}{\beta}$

FOR CIRCULAR WAVEGUIDE:

For TE MODE: $\mathrm{Z}_{\mathrm{te}}=\omega \mu / \beta$

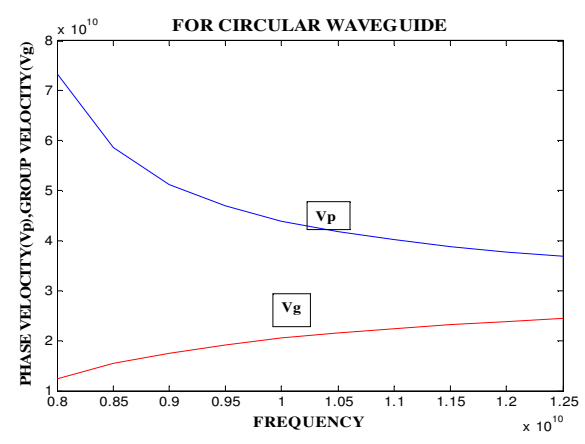

Figure 12. Phase velocity (Circular)

For TM MODE: $Z_{T M}=\frac{\beta \eta}{k}$

For TM MODE: $\mathrm{Z}_{\mathrm{tm}}=\beta / \mu \omega$ 
International Journal of Distributed and Parallel Systems (IJDPS) Vol.3, No.4, July 2012

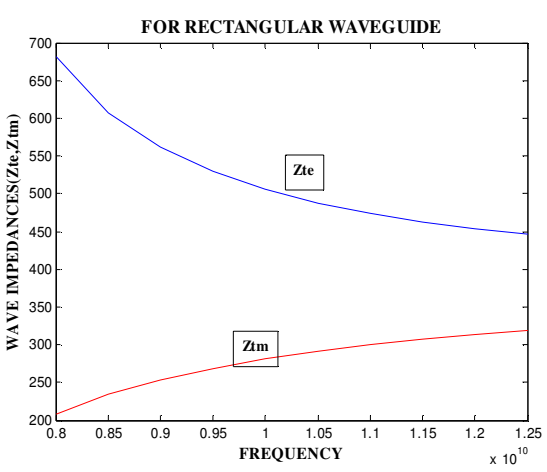

Figure 13. For rectangular

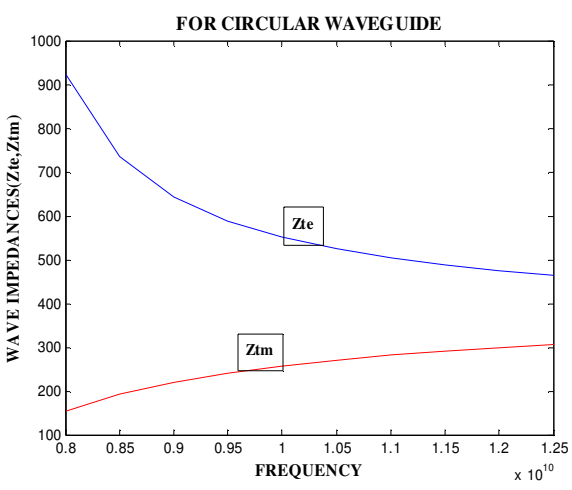

Figure 14. For circular

\subsection{Power in Waveguides}

Electromagnetic energy transmitted into space consists of electric and magnetic fields that are at right angles (90 degrees) to each other and at right angles to the direction of propagation.To establish the relationship this paper use "Poynting vector" theorem.It indicates that a screw(right-hand thread) with its axis perpendicular to the electric and magnetic fields will advance in the direction of propagation if the $\mathrm{E}$ field is rotated to the right (toward the $\mathrm{H}$ field)[9].

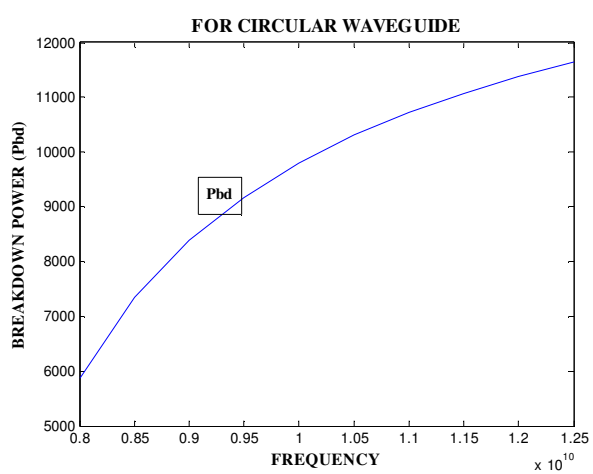

Figure 15.Breakdown power for circular

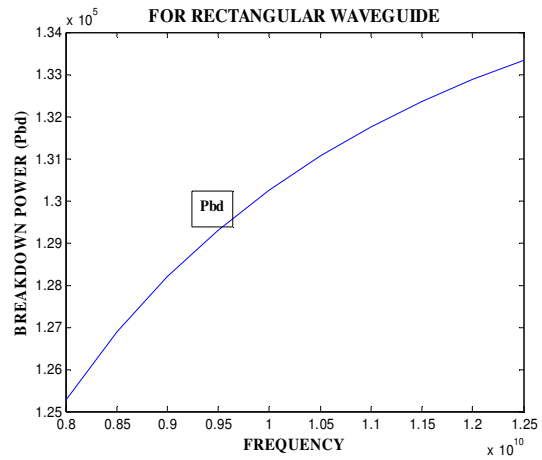

Figure 16.Breakdown power for rectangular

\subsection{Attenuation}

Waveguides can support travelling waves at frequencies higher than their cutoff frequencies. However, the travelling waves are attenuated by losses in the dielectric medium that fills a waveguide and by losses in the conducting sidewalls. Radiation and Induction Losses are similar in that both are caused by the fields surrounding the conductors.Losses occur because some magnetic lines of force about a conductor do not return to the conductor when the cycle alternates.These lines of force are projected into space as radiation and this result in power losses.In waveguide,current flows only along the inside surface of the waveguide, but remember that waveguides have four solid walls to carry the current.So the current-carrying area is large in waveguide but it is small in wire.The current carrying area in waveguide has some little amount of resistance,so due to skin effect losses are very low. 
International Journal of Distributed and Parallel Systems (IJDPS) Vol.3, No.4, July 2012

At the frequencies below the cut off frequency $(\mathrm{f}<\mathrm{fc})$, the propagation constant ' $\gamma$ ' will have only the attenuation term ' $\alpha$ ' that is the phase constant will be the imaginary part.

The cut off attenuation is $\alpha=54.6 / \lambda \mathrm{c}\left(\sqrt{ } 1-(\mathrm{f} / \mathrm{fc})^{\wedge} 2\right)$.

For $\mathrm{f}>\mathrm{fc}$, the waveguide exhibits very low loss and for $\mathrm{f}<\mathrm{fc}$, the attenuation is high and results in full reflection of the wave.Similar to rectangular waveguides it is possible to determine the attenuation in circular waveguide for TE \&TM modes. The attenuation in an airfilled circular waveguide is due to infinite conductivity of the guide walls and is given by;

$\alpha=$ power loss/unit length/(2x(average power transmitted $))$

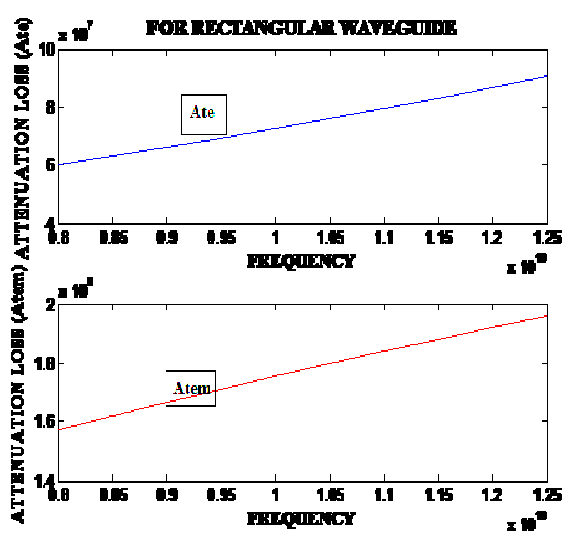

Figure 17. Attenuation(Rectangular)



Figure 18. Attenuation(Circular)

\subsection{VSWR}

VSWR (Voltage Standing Wave Ratio also called Vertical Standing Wave Ratio), is a measure of how efficiently radio-frequency power is transmitted from a power source, through a transmission line, into a load.VSWR is the voltage ratio of the signal on the transmission line:

$\mathrm{VSWR}=|\mathrm{V}(\max )| /|\mathrm{V}(\min )|$

Where $\mathrm{V}(\max )$ is the maximum voltage of the signal along the line, and $\mathrm{V}(\min )$ is the minimum voltage along the line.The SWR is usually defined as a voltage ratio called the VSWR, for voltage standing wave ratio. For example, the VSWR value 1.2:1 denotes maximum standing wave amplitude that is 1.2 times greater than the minimum standing wave value[1].

\subsubsection{Relationship to the reflection coefficient}

The standing wave in a constant transmission line consists some voltage components like forward wave which is superimposed on the reflected wave having the amplitude ' $\mathrm{V}_{\mathrm{f}}$ ' and ' $\mathrm{V}_{\mathrm{r}}$ ' respectively.If the property of transmission line is terminated than its characteristic impedance,then the reflection coefficient $\Gamma$ of these lines are defined as $\Gamma=\frac{V_{r}}{V_{f}}$ where the 
International Journal of Distributed and Parallel Systems (IJDPS) Vol.3, No.4, July 2012

value of $\Gamma$ is a complex number which describes the magnitude as well as the phase shift of the reflection.

$\Gamma=-1$ : maximum negative reflection, when the line is short-circuited.

$\Gamma=0:$ no reflection, when the line is perfectly matched.

$\Gamma=+1$ : maximum positive reflection, when the line is open-circuited.

At some points along the line the two waves interfere constructively, and the resulting amplitude $\mathrm{V}_{\max }$ is the sum of their amplitudes:

$V_{\text {max }}=V_{f}+V_{r}=V_{f}+\rho V_{f}=V_{f}(1+\rho)$

At other points, the waves interfere destructively, and the resulting amplitude $\mathrm{V}_{\min }$ is the difference between their amplitudes:

$V_{\min }=V_{f}-V_{r}=V_{f}-\rho V_{f}=V_{f}(1-\rho)$

The voltage standing wave ratio is then equal to: $V S W R=\frac{V_{\max }}{V_{\min }}=\frac{1+\rho}{1-\rho}$

As $\rho$, the magnitude of $\Gamma$, always falls in the range [0,1] the VSWR is always $\geq+1$.

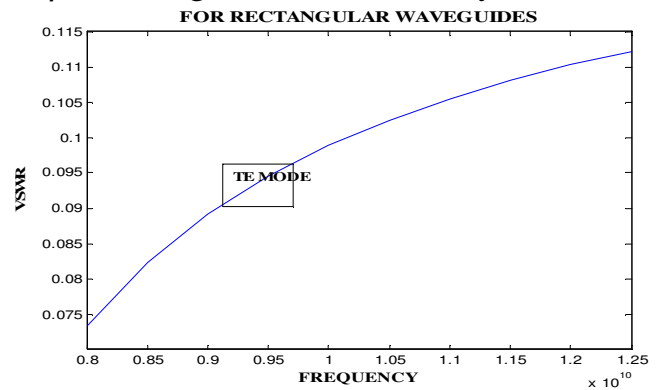

Figure 19. Rectangular TE mode

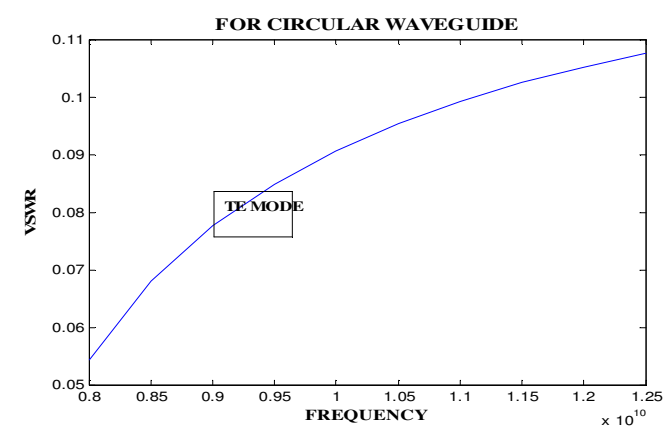

Figure 21. Circular TE mode

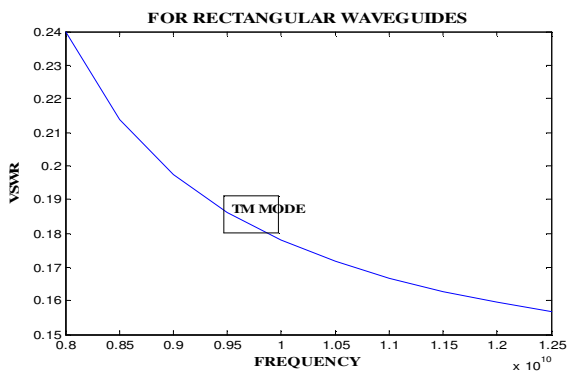

Figure 20. Rectangular TM mode

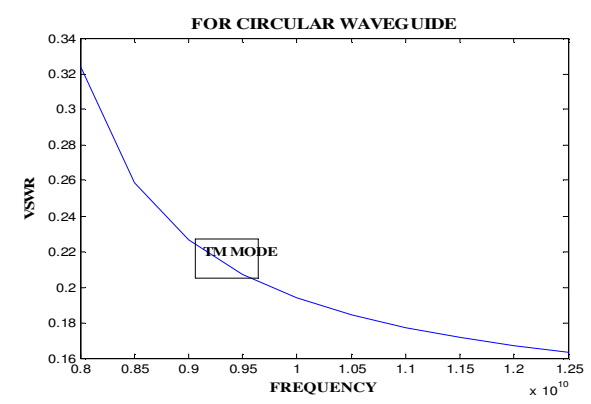

Figure 22.Circular TM mode 
International Journal of Distributed and Parallel Systems (IJDPS) Vol.3, No.4, July 2012

\section{CONCLUSION}

All the above results have been done in Matlab 7.14 version.The parameters of rectangular waveguide and circular waveguide has been verified with the simulated results in Matlab,this paper use to analyze the parameters of waveguides like as (Cut off frequency, Cut off wavelength,Guide wavelength,Phase velocity, Group velocity, Impedance, power, Attenuation, VSWR) and compare their results with the help of output figures so it is easy to analyze from the above results that rectangular waveguide is much better than the circular waveguide.These parameters of Rectangular waveguide showing that the guided wavelength is high,less power required,cutoff wavelength is high but attenuation is more in comparision of circular waveguide.So with the help of these parameters it is easy to select the best waveguide for the applications like in communications field and in military purposes.

\section{REFERENCES}

[1] D.M.Pozar ,2005, "Microwave Engineering” John Wiley and Sons.

[2] Karle S. Packard "The Origin of Waveguides: A Case of Multiple Rediscovery" IEEE transactions on Microwave theory and techniques, vol. mtt-32, no.9, september 1984.

[3] Perregrini Y. Cassivi and P.Arcioni,2002 "Dispersion Characteristics of Substrate Integrated Rectangular Waveguide" IEEE transaction of Microwave and Wireless components, Vol 12,No.9,page no.333-335.

[4] A.Munir and Mohammad F.Y.Musthofa,"Rectangular to Circular Waveguide Converter for Microwave Devices Characterization",International Journal on Electrical Engineering and Informatics, Vol.3,No.3,2011.

[5] Vivek Yadav , Udaybin Singh and A.K.Sinha, 2010 "Analysis and Design of Broadband Square to Circular Waveguide Transitions",International Journal of Microwave and Optical Technology, Vol.5,No.3,May.

[6] R.Vacek, Dec 1998,"Electromagnetic Wave Propagation in General Multimode Waveguide Structures Exhibiting Simultaneously Attenuation,Dispersion and Coupling Phenomena”, Journal of Microwave and Optoelectronics,Vol.1,No.3.

[7] Amos Gilat, Edition-2004, "MATLAB- An Introduction with Applications", John Wiley \& Sons.

[8] Rudra Pratap, 2006 “Getting Started with MATLAB7”. Oxford University Press.

[9] Wikipedia.

[10 Boremann,Amari and Vahldieck Mar 1999 "Analysis and design of circular ridged waveguide components" " IEEE transactions on microwave devices, Volume 47,Issue:3,pages :330-335.

\section{BIOGRAPHY}

Azhar Shadab received his B.E degree in Electronics and communication Engineering from NRI-IIST BHOPAL in 2010, and his M Tech degree in Electronics and Communications Engineering from the Jaypee University of Information Technology, Waknaghat, Himachal Pradesh 2012. His research interests are in the areas of Microwave, VLSI and Signal Processing. Presently he is working on designing of low power fast fourier transform in VLSI.

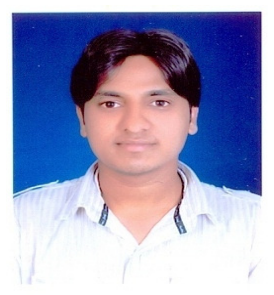


International Journal of Distributed and Parallel Systems (IJDPS) Vol.3, No.4, July 2012

Lokesh kumar received the B.E. degree from RITEE,Raipur(C.G).He is currently pursuing MTECH from Jaypee University of Information Technology, solan. Himachal Pradesh.His research interests are in the VLSI design and circuit.

Mohit kumar has done his B.E from D.Y.Patil College of Engineering (Pune university). He is currently persuing $\mathrm{M}-\mathrm{Tech}\left(2^{\text {nd }} \mathrm{yr}\right)$ from Jaypee University of Information Technology,Solan. Himachal Pradesh

Kamal kishor received his B.E degree in Electronics and communication Engineering from NRI-IIST BHOPAL, 2010, and pursuing $M$ Tech degree in Electronics and Communications Engineering from the Jaypee University of Information Technology, Waknaghat, Himachal Pradesh. His research interests are in the areas of Microwave \& Antenna.

Akash Sethi received his Bachelor of Engineering degree in Electronics and Communications Engineering from G.G.I.T.S. Jabalpur(M.P.) 2009, and M.Tech degree in Electronics and Communications Engineering from the Jaypee University of Information Technology, Waknaghat(H.P.) 2012. His research interests are in the areas of Wireless Communication, Electrical Circuit Analysis and VLSI.

Ila Sharma received her B.E degree in Electronics and Telecommunications Engineering fromG.E.C.Raipur, Chhattisgarh 2009, and her M Tech degree in Electronics and Communications Engineering from the Jaypee University of Information Technology, Waknaghat, Himachal Pradesh 2012. Her research interests are in the areas of Wireless Communication, Analog Electronics and Signal Processing.
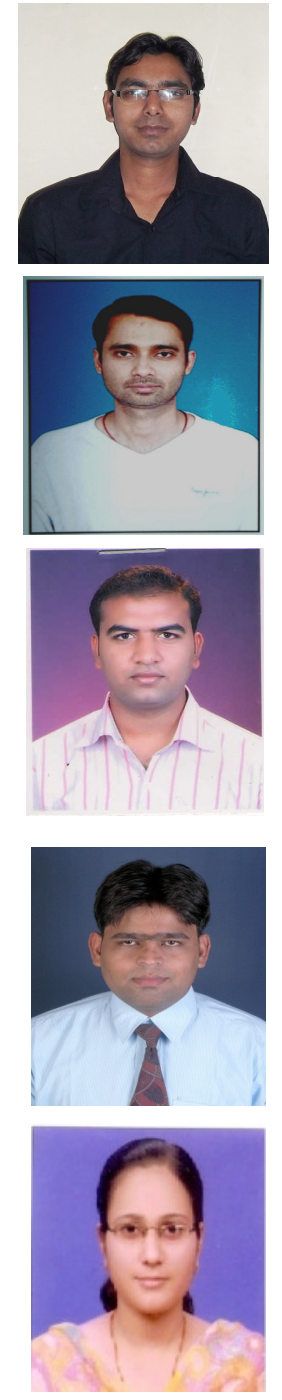